\title{
DICOM Study
}

National Cancer Institute

\section{Source}

National Cancer Institute. DICOM Study. NCI Thesaurus. Code C63537.

One or more series of images taken on a single visit to a medical facility. 